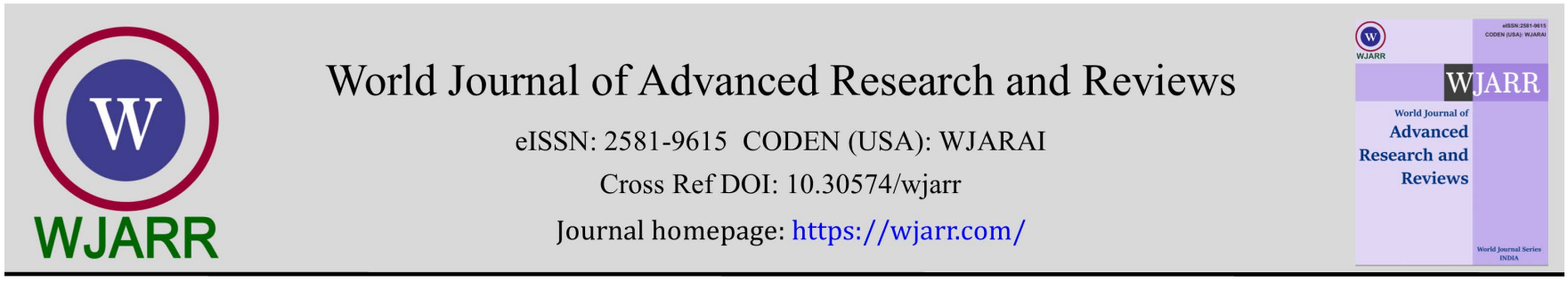

(CASE STUdY)

Check for updates

\title{
Continuous renal replacement therapy and ECMO in COVID patients, a Moroccan case report experience
}

\author{
Younes Oujidi 1, ${ }^{*}$, Imane Melhaoui ${ }^{2}$, Layla Kherroubi ${ }^{3}$, Houssam Bkiyar ${ }^{1}$ and Brahim Housni 1 \\ ${ }^{1}$ Anaesthesia and Resuscitation Service - Hospital University Mohammed VI of Oujda. Oujda Faculty of Medicine and \\ Pharmacy, Mohammed Premier University, Oujda, Morocco. \\ 2 Nephrology Department - Hospital University Mohammed VI of Oujda. Oujda Faculty of Medicine and Pharmacy, \\ Mohammed Premier University, Oujda, Morocco. \\ ${ }^{3}$ Internal medicine Department - Hospital University Mohammed VI of Oujda. Oujda Faculty of Medicine and Pharmacy, \\ Mohammed Premier University, Oujda, Morocco.
}

World Journal of Advanced Research and Reviews, 2021, 09(02), 159-162

Publication history: Received on 15 January 2021; revised on 17 February 2021; accepted on 19 February 2021

Article DOI: https://doi.org/10.30574/wjarr.2021.9.2.0059

\begin{abstract}
Introduction: Extracorporeal membrane oxygenation (ECMO) is a therapy that ameliorate the oxygenation of hypoxemia refractory patient it could be associated to a kidney failure that necessity a Continuous Renal Replacement Therapy.

Case Report: We report the case of a 68-year-old patient, who presents ARDS due to covid infection, during his hospitalisation the patient presented a refractory hypoxemia with the need to set up ECMO, the case worsened with kidney failure with need for CRRT.

Conclusion: The association between ECMO and CRRT might be a safe and effective technique. A variety of ECMO and CRRT combination methods can be chosen, this remains an association that should be investigated in order to improve the prognosis of kidney failure on ECMO.
\end{abstract}

Keywords: COVID; ECMO; CRRT; SDRA

\section{Introduction}

Extracorporeal membrane oxygenation (ECMO) is a therapy that improves oxygenation in patients with acute cardiac or pulmonary dysfunctions with a high risk of developing acute kidney injury and fluid overload. Continuous Renal Replacement Therapy (CRRT) can provide kidney replacement and fluid management. The association of the two therapies is still not well codified. We report the case of a Patient who has presented with a severe SDRA requiring an ECMO, complicated with acute kidney injury needed for a CRRT.

\section{Cases Report}

We report the case of a 68-year-old patient, having hypertension under treatment as an ATCD, admitted for management of respiratory distress following an infection with SARS COV 2, the clinical examination in the intensive unit care on admission found a conscious patient GCS 15/15, Saturation was $60 \%$ ambient air , hemodynamic stable,

\footnotetext{
${ }^{*}$ Corresponding author: Younes Oujidi

Anaesthesia and Resuscitation Service - Hospital University Mohammed VI of Oujda. Oujda Faculty of Medicine and Pharmacy, Mohammed Premier University, Oujda, Morocco.

Copyright (@ 2021 Author(s) retain the copyright of this article. This article is published under the terms of the Creative Commons Attribution Liscense 4.0.
} 
febrile at 38.5C. biological balance finds lymphopenia with elevated LDH and Ferretinemie levels, blood gaz : P02 to $70 \mathrm{mmhg}$ under optiflow (oxygen flow at 701/min and FIO2 at 100\%), Chest CT with 75\% CORADS 6 (Figure 1),put under hydroxychloroquine, azithromycin , dexamethasone $8 \mathrm{mg} / \mathrm{j}$, enoxaparin $0.6 \mathrm{ui} / 12$, 3erd generation cephalosporins $2 \mathrm{~g} / \mathrm{j}$ and ciprofloxacin $200 \mathrm{mg} / 12 \mathrm{~h}$, Paracetamole, Vitamine C et Zinc, at 2end day of his admission the patient presented an aggravation of his state needed to be intubated, with a baby lung ventilation parametre (vt a 420 ml, Fr a 20, PEEP a 10 , FiO2 a 100\%) with a hemodynamique need support by the norépinéphrine $(1 \mathrm{mg} / \mathrm{h})$, the blood gaz post intubation found ,a ratio of $\mathrm{PaO} 2 /$ Fio2 at 70 with metabolic acidosis, a chest X-ray made: white lung in bilateral (figure 2), subsequently, the patient did not present an improvement in his respiratory status, even though he placed it in Decubitus ventral with a PaO2/FiO2 ratio always less than 100 .the patient was put on ECMO VV ( femoral and right jugular ) on day 4 of his hospitalization and J2 of his intubation. Under ECMO the PaO2/FiO2 ratio improved to 180, at j6 the patient presented a septic shock requiring an increase in doses of noradrenaline arriving $5 \mathrm{mg} / \mathrm{h}$ complicated of an acute renal failure class Kadigo 3 (anuria of 15 hours with DFG at $30 \mathrm{ml} / \mathrm{min} / 1.73 \mathrm{~m} 2$ ) A blood gas shows a pH at 6.89, PO2 at $150 \mathrm{mmhg}$; PCO2 at $35 \mathrm{mmhg}$, HCO3 $-6.5 \mathrm{mEq} / \mathrm{l}$. the patient was dialysis by a continuous hemofiltration CVVH (figure 1) by inclusion of a CRRT device in the ECMO circuit as well as put on piperacillin tazobactam amikacin and fluconazol , At 28h of continuous hemofiltration the patient still presents a worsening of his hemodynamic state and died at $48 \mathrm{~h}$ after his connection to hemofiltration.

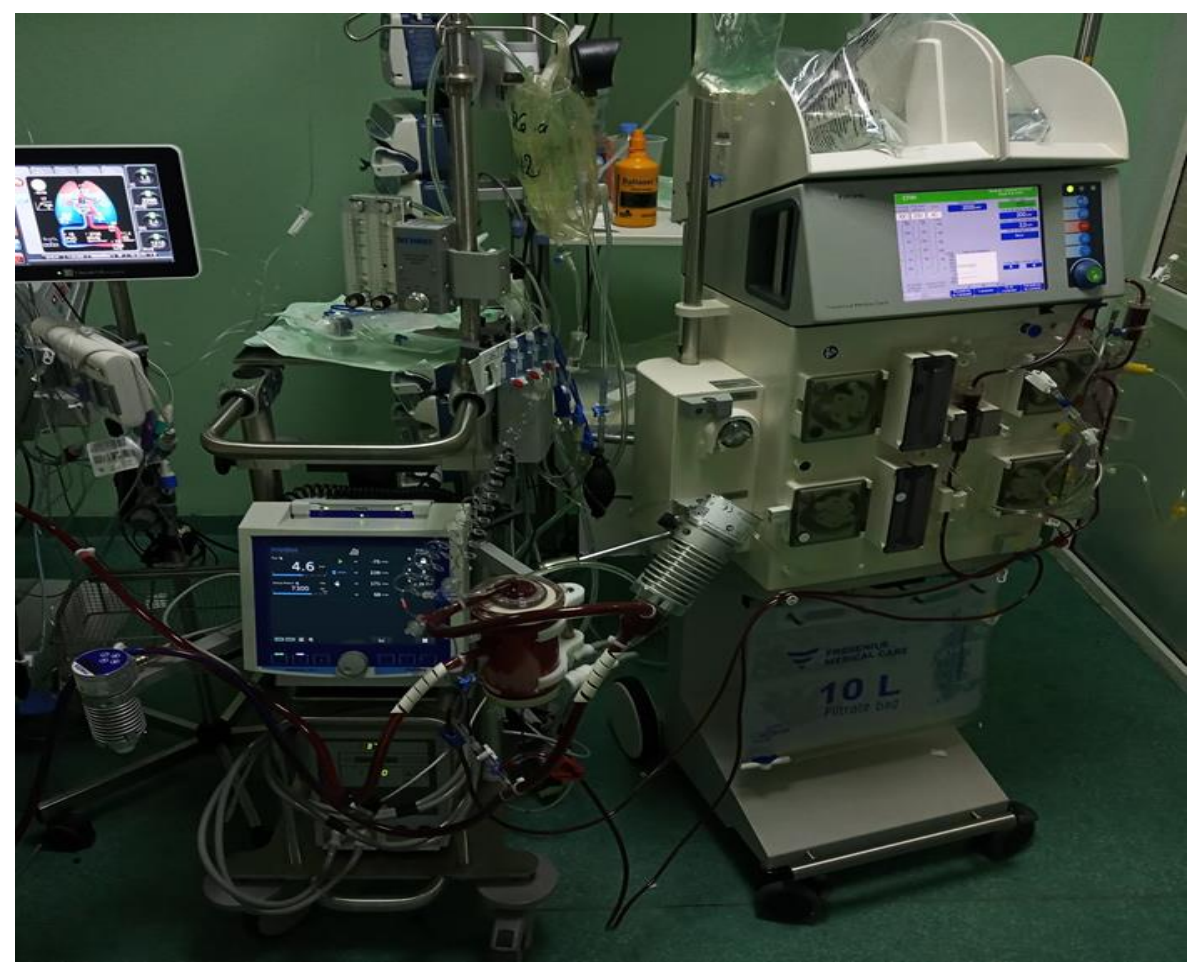

Figure 1 Patient dialysis by a continuous hemofiltration CVVH, by inclusion of a CRRT device in the ECMO circuit.

\section{Discussion}

The use of venous-venous extracorporeal membrane oxygenation (VV-ECMO) due to the virus SARS-CoV-2 and the clinical disease Covid-19 in the management of acute respiratory distress syndrome (ARDS) can improve gas exchange and may reduce Lung injury caused by ventilator [1] ,A study made by the A.combes team showed that in a trial involving patients with very severe ARDS, an analysis of the primary endpoint (60-day mortality) showed that early ECMO had no obvious benefit compared with traditional mechanical ventilation strategies (including cross-use ECMO) (28\% of patients in the control group used it) [2]. As the life support for patients with cardiopulmonary failure, ECMO is a long-term invasive respiratory and circulatory assistance system. During ECMO treatment, there are a variety of risk factors that may lead to adverse complications, such as long-term implantation of artificial devices, nonphysiological interventions on the respiratory and circulatory systems, and the patient's pathophysiological condition[3].The mechanical-related complications of the ECMO system are caused by mechanical equipment dysfunction, including thrombosis, intubation problems, oxygenator dysfunction, and air embolism. We also found that bleeding, renal insufficiency, infection, hemolysis, hyperbilirubinemia, and certain circulatory, respiratory and neurological complications are non-mechanical complications. In addition, patients receiving ECMO treatment usually 
suffer from drug-resistant bacterial infections [4]. There are many methods to perform CRRT during ECMO, which can be divided into three main methods: RRT is performed through venous access that has nothing to do with the ECMO circuit, introduction of a hemofiltration filter into the ECMO circuit using intravenous infusion pumps to control the ultrafiltrate volume and inclusion of a CRRT device in the ECMO circuit [5] (figure 2).

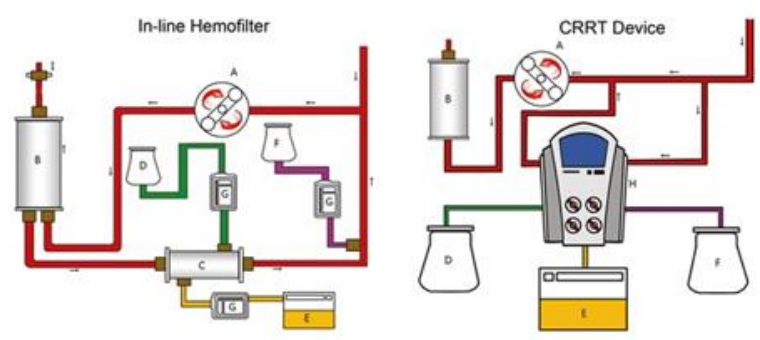
Two typical methods of combining $E C M O$ and the $C R R T$ circuit. $A=E C M O$ pump; $B=$ Oxygenator; $C=$ Hemofilter; $D=$ Dialysis
fluid; $E=$ Effluent fluid; $F=$ Replacement fluid; $G=$ Intravenous pump; $H=C R R T$ device; $I=$ Ultrasonic flow probe. $C R R T$, continuous
renal replacement therapy; ECMO, extracorporeal membrane oxygenation.

Figure 2 Methods of combining ECMO and the CRRT circuit

For our case, the staff chose ECMO-V V because the patient was on a Refractory hypoxemia due to damage of the respiratory system alone, the evolution was marked by a state of septic shock complicated renal failure (Kadigo 3) which needed a continuous renal replacement therapy (CRRT) by inclusion of a CRRT device in the ECMO circuit.in letterature a higher mortality rate was observed after data analysis, which indicates that the requirement for renal placement therapy could be the risk factor for mortality, A recently published paper by Luo et al. [6] showed that AKI is associated with in hospital mortality in critically ill patients, no matter if it was defined by RIFLE, AKIN or KDIGO criteria. The adverse outcome is related to the increased severity of AKI. In populations needing ECMO, a similar situation was observed [6-7]. In a cohort study by Zwiers et al. [8] using RIFLE criteria, two thirds of neonates receiving ECMO had $\mathrm{AKI}$, and the mortality risk in the Failure category was significantly increased. We tried to assess the difference in AKI between ECMO and ECMO plus CRRT groups.

\section{Conclusion}

The association between ECMO and CRRT might be a safe and effective technique that improves fluid balance and ameliorates electrolyte disturbances. A variety of ECMO and CRRT combination methods can be chosen depending on the experience of the teams, and despite the mortality rate during this combination, this remains an association that should be investigated in order to improve the prognosis of kidney failure on ECMO.

\section{Compliance with ethical standards}

\section{Disclosure of conflict of interest}

This research did not receive specific grants from funding agencies in the public, commercial or not-for-profit sectors.

\section{Statement of informed consent}

Informed consent was obtained from all individual participants included in the study.

\section{References}

[1] Marguerite M, Hoyler MD, Shreyajit Kumar MD, Richard Thalappillil MD, Robert S. White, MD, MS, Christopher W, Tam MD . VV-ECMO usage in ARDS due to COVID-19: Clinical, practical and ethical considerations. J Clin Anesth. 2020 Oct; 65: 109893.

[2] Combes A, Hajage D, Capellier G, Demoule A, Lavoue S, Guervilly C. Extracorporeal membrane oxygenation for severe acute respiratory distress syndrome. N Engl J Med. 2018; 378: 1965-1975.

[3] Millar JE, Fanning JP, McDonald CI, et al. The inflammatory response to extracorporeal membrane oxygenation (ECMO): A review of pathophysiology. Crit Care. 2016; 20(1): 387. 
[4] Ma X, Liang M, Ding M, Liu W, Ma H, Zhou X, Ren H. Extracorporeal Membrane Oxygenation (ECMO) in Critically Ill Patients with Coronavirus Disease 2019 (COVID-19) Pneumonia and Acute Respiratory Distress Syndrome (ARDS).Med Sci Monit. 2020 Aug 6; 26: e925364.

[5] Han Chen, Rong-Guo Yu, Ning-Ning Yin, Jian-Xin Zhou. Combination of extracorporeal membrane oxygenation and continuous renal replacement therapy in critically ill patients: a systematic review.Crit Care. 2014; 18(6): 675.

[6] Luo X, Jiang L, Du B, Wen Y, Wang M, Xi X. A comparison of different diagnostic criteria of acute kidney injury in critically ill patients. Crit Care. 2014; 18: R144.

[7] Askenazi DJ, Ambalavanan N, Hamilton K, Cutter G, Laney D, Kaslow R, Georgeson K, Barnhart DC, Dimmitt RA. Acute kidney injury and renal replacement therapy independently predict mortality in neonatal and pediatric non cardiac patients on extracorporeal membrane oxygenation. Pediatr Crit Care Med. 2011; 12: e1-e6.

[8] Zwiers AJ, de Wildt SN, Hop WC, Dorresteijn EM, Gischler SJ, Tibboel D, Cransberg K. Acute kidney injury is a frequent complication in critically ill neonates receiving extracorporeal membrane oxygenation: a 14-year cohort study. Crit Care. 2013; 17: R151. 